\title{
Asteroseismology in action: a test of spin-orbit synchronism in the close binary system Feige 48
}

\author{
V. Van Grootel ${ }^{1,2}$, S. Charpinet ${ }^{1}$, G. Fontaine ${ }^{2}$, and P. Brassard ${ }^{2}$ \\ ${ }^{1}$ Laboratoire d'Astrophysique de Toulouse-Tarbes, Université de Toulouse, CNRS, 14 av. E. Belin, 31400 Toulouse, France \\ e-mail: [valerie.vangrootel; stephane.charpinet]@ast.obs-mip.fr \\ 2 Département de Physique, Université de Montréal, CP 6128, Succursale Centre-Ville, Montréal, QC H3C 3J7, Canada \\ e-mail: [fontaine; brassard]@astro.umontreal.ca
}

Received 11 February 2008 / Accepted 18 March 2008

ABSTRACT

\begin{abstract}
Aims. In this paper, we provide a test of spin-orbit synchronism in the close binary system Feige 48 . This system is made of a rapidly pulsating subdwarf B (sdB) star with an unseen companion, most likely a white dwarf. The presence of nonradial oscillations offers the opportunity to infer the inner profile and period of rotation of the primary star through asteroseismology. This constitutes the key element for testing spin-orbit synchronism in depth, since stellar internal layers are inaccessible to traditional techniques.

Methods. We carried out a new asteroseismic analysis of Feige 48 following the so-called "forward modeling" approach. This is done with our latest optimization algorithms, which have been updated to incorporate the effect of stellar rotation on pulsations, assuming various internal rotation laws. In this analysis, the simultaneous match of all the frequencies observed in Feige 48 leads objectively to the full identification of the pulsation modes through the determination of the indices $k, \ell, m$. It also leads to determining the structural and rotational parameters of Feige 48.

Results. Our optimal model, obtained with a solid-body rotation law, is characterized by a spin period of $9.028 \pm 0.480 \mathrm{~h}$. This value is remarkably similar to the system's orbital period of $9.024 \pm 0.072 \mathrm{~h}$, measured independently from radial velocity variations. We further demonstrate that the hypothesis of differential rotation of the core - including a fast rotating core - must be eliminated for Feige 48.

Conclusions. These results strongly imply that Feige 48 rotates as a solid body in a tidally locked system. This constitutes the first explicit demonstration of spin-orbit synchronism in a binary star by asteroseismic means.
\end{abstract}

Key words. stars: binaries: close - stars: oscillations - stars: rotation - stars: subdwarfs - stars: individual: Feige 48

\section{Introduction}

Close binary stars have always played a crucial role in astrophysics. They not only represent at least half of all stars, they also provide stringent tests of stellar evolution models (see, e.g., Hilditch 2001). In the light of asteroseismology (i.e., the study of the structure of stars through the interpretation of their oscillation modes), pulsating stars in close binary systems become laboratories where binary evolution scenarios can be tested in terms, for example, of energy loss or angular momentum transport through internal rotation. Considering that our current knowledge of stars relies almost exclusively on what is seen from their surface, the promise of extracting direct information on their internal structure through asteroseismology has tremendous implications.

Among all known classes of pulsating stars spread over the Hertzsprung-Russell diagram, the group of rapidly pulsating hot subdwarf B (sdB) stars (or EC 14026 stars) has proved its potential for performing objective asteroseismic analyses (see, e.g., Fontaine et al. 2006a, for a recent review of this). The sdB stars are core-helium burning objects with masses around $0.5 M_{\odot}$ surrounded by very thin hydrogen-rich envelopes $\left(M_{\text {env }}<0.02 M_{\odot}\right)$. With effective temperatures between 20000 and $40000 \mathrm{~K}$ and surface gravities $\log g$ in the range 5.0-6.2, they form the very hot end of the horizontal branch, the so-called extreme horizontal branch (EHB; Heber 1986). Seldom known to many astronomers, subdwarf B stars dominate the population of faint blue objects down to $V \sim 16$, and are found both in the old disk (field stars) and halo population (globular cluster members) of our Galaxy. They are believed to be the main source of the UV excess (the so-called UV-upturn phenomenon) observed in elliptical galaxies (Brown et al. 1997), and might well hold the key to a secure age estimate for some of the oldest structures in the Universe.

Understanding the formation of sdB stars is one of the remaining challenges related to stellar evolution theory. While it is generally accepted that they descend from the Red Giant Branch (RGB), the difficulties lie in understanding in detail how they are formed. One important clue, however, is the fact that the fraction of $\mathrm{sdB}$ stars in short period binaries (with orbital periods $\lesssim 10$ days) is found to be high. Indeed, Green et al. (1997) found a fraction of more than $50 \%$ in their sample of bright field subdwarfs observed at the MMT. For their part, Maxted et al. (2001) found that $\sim 65 \%$ of their sample from the PalomarGreen survey were such binaries, whereas somewhat lower fractions of $\sim 40 \%$ were found recently for the SPY (Supernova Ia Progenitor Survey) sample (Napiwotzki et al. 2004) and 48\% for yet another sample drawn from the Edinburgh-Cape cata$\log$ (Morales-Rueda et al. 2006). Another non-negligible fraction of sdB stars resides in long period binaries (with periods of hundreds to thousands of days) having main sequence companions (Saffer et al. 2001). In this context, several channels 
for the formation of $\mathrm{sdB}$ stars were considered by Han et al. (2002, 2003) to build synthetic binary population simulations. These include evolution through a common envelope (CE), stable Roche lobe overflow (RLOF), and the merger of two helium white dwarfs. These distinct scenarios give different mass and hydrogen-envelope thickness distributions for the resulting sdB stars. In a nutshell, CE evolution would produce sdB's in close binary systems, while longer period binaries would result from RLOF, and the merger channel could explain a fraction of the population of single stars. The latter ones could also result from single star evolution, by enhanced and tuned mass loss at the tip of the RGB (D'Cruz et al. 1996). After corehelium exhaustion, $\mathrm{sdB}$ stars evolve directly toward the white dwarf cooling sequence, without experiencing the ascend of the Asymptotic Giant Branch (AGB) and Planetary Nebulae (PN) phase of usual stellar evolution (Dorman et al. 1993).

Interest in sdB stars has increased spectacularly in recent years following the discovery of rapid oscillations in some of them (Kilkenny et al. 1997; and see also Charpinet et al. 1996, 1997). A small fraction ( $5 \%)$ of the known sdB stars exhibit rapid, multi-periodic luminosity variations over a few hundreds of seconds, thus giving the opportunity of using asteroseismology to determine their structural parameters and test stellar formation and evolution theory. The presence of excited pulsation modes, usually low-degree, low-order $p$-modes, is well understood by the action of a classic $\kappa$-effect associated with an opacity bump due to partial ionization of heavy metals - especially iron - locally enhanced by radiative levitation at work in the envelope of these stars (Charpinet et al. 2001). Beyond iron, the importance of nickel on the opacity profile, as well as the opacity sources themselves, have been recently underlined by Jeffery \& Saio $(2006,2007)$. The potential of sdB stars for asteroseismology has been exploited over several years now, and has so far resulted in the asteroseismic determination of the structural parameters for seven EC 14026 pulsators: PG 0014+067 (Brassard et al. 2001), PG 1047+003 (Charpinet et al. 2003), PG 1219+534 (Charpinet et al. 2005b), Feige 48 (Charpinet et al. 2005a), EC 20117-4014 (Randall et al. 2006), PG 1325+101 (Charpinet et al. 2006), and PG 0911+546 (Randall et al. 2007). These first asteroseismic results have shown promising trends such as matching the expected mass and hydrogen-envelope mass distributions (see, e.g., Fontaine et al. 2006a).

In parallel, we have pursued the improvement of our codes and models. For instance, the effects of stellar rotation on pulsation modes was recently incorporated in the procedure used for performing asteroseismic analyses. This improvement was deemed necessary in those few cases where rotation bears an obvious and important signature on the observed frequency spectrum through rotational splitting. As a first application of this improved modeling approach, we present, in this paper, a new asteroseismic analysis of the moderately rotating pulsator Feige $48\left(\mathrm{~V}^{*} \mathrm{KL}\right.$ UMa), one of the best-studied sdB stars. It belongs to a fairly close binary system with a faint unseen companion (most likely a white dwarf). The system has a measured orbital period of $9.024 \pm 0.072 \mathrm{~h}$ (O'Toole et al. 2004). As a pulsating star showing rotational splitting, we singled out Feige 48 as an ideal target to test the concept of spin-orbit synchronism in a close binary system.

Theoretical frameworks on tidal friction in close binaries have been developed essentially by Zahn and Tassoul \& Tassoul (Zahn 1977; Tassoul \& Tassoul 1992, and references therein). These studies show that synchronization times depend notably on the orbital period, but can differ by orders of magnitude depending on the physical mechanism invoked to explain synchronism. This is particularly true in the case of hot stars with radiative envelopes (such as sdB stars), where tidal forces are believed to be less efficient for synchronization. While the mechanism of Zahn is not dissipative enough to explain the observed levels of synchronization, the question of the validity of the mechanism proposed by Tassoul \& Tassoul is still under debate, given its free parameter dependence (Claret et al. 1995; Claret \& Cunha 1997, and references therein). Nevertheless, it is expected that very close $\mathrm{sdB}+$ white dwarf binaries such as KPD 0422+5421 (Orosz \& Wade 1999) and KPD 1930+2752 (Billères et al. 2000; Maxted et al. 2000) with an orbital period of, respectively, $2.16 \mathrm{~h}$ and $2.28 \mathrm{~h}$, should have achieved spin-orbit synchronism over the typical lifetime of a sdB star $\left(\sim 10^{8} \mathrm{yrs}\right)$. And indeed, both systems show an ellipsoidal luminosity variation with a period equal to exactly half of the orbital period, as expected in tidally locked systems. The hypothesis of spin-orbit synchronism was indeed used by Geier et al. (2007) to constrain the binary parameters of KPD $1930+2752$. In a recent interesting development, Geier et al. (2008) presented another study of a third sdB + white dwarf binary system, PG 0101+039. By combining accurate spectroscopic measurements with ellipsoidal light curve modeling, they were able to show that the tiny ellipsoidal variation observed in PG $0101+039$ has a period that is, once again, equal to half the orbital period of $13.68 \mathrm{~h}$. These results suggest rather strongly that tidal synchronization is likely achieved in $\mathrm{sdB}+$ white dwarf binaries with orbital periods of less than $\sim 14 \mathrm{~h}$ in general. We point out, however, that observations and modeling of the ellipsoidal variation deal only with the atmospheric layers, and that a much more stringent test of spin-orbit synchronism would involve the internal rotation profile of the star. The latter is a fundamental component in the theory of spin-orbit synchronism, but cannot be inferred through traditional techniques. The fact that the primary component of the Feige 48 system is a rapidly oscillating star offers the exciting possibility of using asteroseismology to test the idea of synchronous rotation in the system.

In Sect. 2, we propose a short review of the known properties of Feige 48, including results from a former asteroseismic study carried out by Charpinet et al. (2005a) that did not incorporate the effects of rotation on the pulsation periods of the $\mathrm{sdB}$ star in the fitting procedure. Our new asteroseismic analysis including, this time, the effects of rotation is reported in Sect. 3. As we implemented various internal rotation laws, it was possible to test several hypotheses, such as solid body and differential core rotation for the sdB star. A comparison with the previous seismic work on Feige 48 is then presented in Sect. 4, followed by a discussion on the implications of our results in Sect. 5. A summary and conclusions are finally provided in Sect. 6.

\section{Known properties of Feige $\mathbf{4 8}$}

\subsection{From spectroscopy}

The first spectroscopic estimates for the atmospheric parameters of Feige 48 were given by Koen et al. (1998) using a spectrum obtained with the intermediate-dispersion spectrograph at the 2.5-m Isaac Newton telescope. Using grids of LTE model atmospheres with zero helium abundance (since no He I line was visible in their spectrum), they derived $T_{\mathrm{eff}}=28900 \pm 300 \mathrm{~K}$ and $\log g=5.45 \pm 0.05$.

Subsequently, Heber et al. (2000) obtained and analyzed a high $\mathrm{S} / \mathrm{N}$ ratio Keck HIRES spectrum of Feige 48, and determined $T_{\mathrm{eff}}=29500 \pm 300 \mathrm{~K}, \log g=5.50 \pm 0.05$ and $\log N(\mathrm{He}) / N(\mathrm{H})=-2.93 \pm 0.05$, using line-blanketed LTE 
and NLTE model atmospheres. It was also possible, from the very sharp metal lines seen in that spectrum, to constrain the projected rotational velocity of Feige 48 by setting a limit of $V \sin i \lesssim 5 \mathrm{~km} \mathrm{~s}^{-1}$. This upper bound has later on been confirmed by O'Toole et al. (2004) from three Fe III lines seen in HST/STIS and archival FUSE spectra. It is also by using these $\mathrm{UV} / \mathrm{FUV}$ spectra that the authors discovered the binary nature of Feige 48 . They were able to measure a velocity semi-amplitude $K=28.0 \pm 0.2 \mathrm{~km} \mathrm{~s}^{-1}$ for the sdB component and estimate the orbital period, $P_{\text {orb }}=9.024 \pm 0.072 \mathrm{~h}$, for the system. They also showed, under the assumption of a tidally locked system and using a representative radius for the $\mathrm{sdB}$ star, that the orbital inclination must be $\lesssim 11.4^{\circ}$ (implying that the sdB star is seen nearly pole-on). This result, combined with the rather small velocity semi-amplitude observed, indicates that the unseen companion is most likely a white dwarf with a mass $\geq 0.46 M_{\odot}$, as derived from the computed mass function $f\left(M_{\mathrm{sdB}}, M_{\mathrm{comp}}\right)$.

In order to obtain further estimates for the atmospheric parameters of Feige 48, additional spectra were obtained by E.M. Green with the blue spectrograph at the 6.5-m MMT and the Steward Observatory 2.3-m telescope. This is part of a large ongoing program aimed at homogeneously determine the surface parameters of sdB stars (further details on this will be provided in Green et al., in preparation). The analysis of these spectra using NLTE $\mathrm{H} / \mathrm{He}$ model atmospheres is reported in Charpinet et al. (2005a), and the values derived in that case are $T_{\text {eff }}=29580 \pm 370 \mathrm{~K}, \log g=5.480 \pm 0.046$, and $\log N(\mathrm{He}) / N(\mathrm{H})=-2.95 \pm 0.08$. These estimates from three independent sources using different spectra and atmospheric models are remarkably consistent with each other and give us confidence in their reliability. They place Feige 48 close to the red edge of the EC 14026 instability region in the $\log g-T_{\text {eff }}$ plane.

Finally, for completeness we mention that the abundances of iron-group elements in the atmosphere of Feige 48 have been accurately determined from UV/FUV spectroscopy obtained with FUSE (Chayer et al. 2004) and HST/STIS (O'Toole \& Heber 2006).

\subsection{From time-series photometry}

Rapid oscillations in the sdB star Feige 48 were first discovered by Koen et al. (1998). On the basis of $\sim 30$ h of white light photometry gathered at the McDonald Observatory 0.9-m telescope, five periods were uncovered in the range $342-380 \mathrm{~s}$. These periods are somewhat longer than in most of the other known EC 14026 stars, but are fully compatible with the position of the star near the red edge of the instability strip. In a follow-up study, Reed et al. (2004) observed Feige 48 over 5 years with small telescopes and confirmed the periods measured by Koen et al. (1998), as summarized in the last column of Table 1.

The present analysis is based on the same observations presented in Charpinet et al. (2005a), namely, $\sim 15.7 \mathrm{~h}$ of high signal-to-noise ratio white light photometry obtained at the Canada-France-Hawaii Telescope (CFHT) during a six-night run scheduled in June 1998 using LAPOUNE, the Montréal portable three-channel photometer. This campaign resulted in a frequency resolution in the Fourier domain of $2.17 \mu \mathrm{Hz}$ and a relatively modest duty cycle of $12.8 \%$. However, its superb sensitivity allowed us to detect extra pulsation frequencies from the light curve of Feige 48 that were not seen previously. The five pulsations detected by Koen et al. (1998) were indeed recovered, and four additional periods could be identified in the CFHT data (see Charpinet et al. 2005a, for details). Table 1 lists the properties of the nine harmonic oscillations detected in Feige 48.
Table 1. Harmonic oscillations detected in the light curve of Feige 48.

\begin{tabular}{cccccc}
\hline \hline ID & $\begin{array}{c}\text { Frequency } \\
(\mathrm{mHz})\end{array}$ & $\begin{array}{c}\text { Period } \\
(\mathrm{s})\end{array}$ & $\begin{array}{c}\text { Amplitude } \\
(\%)\end{array}$ & $\begin{array}{c}\text { Spacing } \\
(\mu \mathrm{Hz})\end{array}$ & $\begin{array}{c}\text { Reed et al. (2004) } \\
\text { Period }(\mathrm{s})\end{array}$ \\
\hline$f_{1}^{-}$ & 2.91522 & 343.027 & 0.071 & +25.0 & $\ldots$ \\
$f_{1}$ & 2.89020 & 345.997 & 0.111 & $\ldots$ & $\ldots$ \\
$f_{2}^{-}$ & 2.90640 & 344.068 & 0.411 & +28.9 & 344.08 \\
$f_{2}$ & 2.87745 & 347.530 & 0.640 & $\ldots$ & 347.56 \\
$f_{2}^{+}$ & 2.85107 & 350.746 & 0.165 & -26.4 & 350.75 \\
$f_{3}$ or $f_{1}^{+}$ & 2.83728 & 352.450 & 0.116 & -52.9 & 352.40 \\
$f_{4}^{-}$ & 2.67180 & 374.280 & 0.039 & +29.5 & $\ldots$ \\
$f_{4}$ & 2.64228 & 378.461 & 0.131 & $\ldots$ & 378.50 \\
$f_{4}^{+}$ & 2.61105 & 382.988 & 0.043 & -31.2 & $\ldots$ \\
\hline
\end{tabular}

Four groups of modes can naturally be constructed from the nine periods uncovered: two triplets $\left(f_{2}^{-}, f_{2}, f_{2}^{+}\right)$and $\left(f_{4}^{-}, f_{4}, f_{4}^{+}\right)$, a doublet $\left(f_{1}^{-}, f_{1}\right)$, and a singlet $\left(f_{3}\right)$. The components of the multiplets are approximately evenly distributed in frequency with a mean spacing of about $\sim 28 \mu \mathrm{Hz}$, as shown in the fifth column of Table 1 . Such nearly symmetric structures can be interpreted as the components of modes with the same $k$ and $\ell$ values, but different $m$ indices bearing the signature of the rotation of the star. This is well known in pulsation theory: rotation lifts the $(2 \ell+1)$-fold mode degeneracy of a spherical star in frequency space. We note that the $352.45 \mathrm{~s}$ period, considered as an independent mode (with the ID $f_{3}$ ) by Charpinet et al. (2005a), is separated from $f_{1}$ by only $52.9 \mu \mathrm{Hz}$. Within the resolution of the data set, this is consistent with twice the mean spacing and, therefore, this period could alternatively be interpreted as a component of the $f_{1}$ complex with $|\Delta m|=2$. This ambiguity is one of the reasons that pushed us to carry out a new asteroseismic analysis of this star using our improved codes incorporating rotational splitting. In this way, a simultaneous fit of all nine frequencies can be carried out without a priori assumptions, contrary to the previous analysis based on four assumed $m=0$ components.

\subsection{Results from the first asteroseismic analysis}

In the asteroseismic analysis without rotation presented by Charpinet et al. (2005a), four periods were selected as the central components of the multiplets. Only those independent modes $\left(f_{1}, f_{2}, f_{3}\right.$, and $f_{4}$ in Table 1$)$ could be used in the comparison with degenerate frequency spectra computed from purely spherical models. This analysis led to the identification of the $(k, \ell)$ indices of the four periods and to the determination of the structural parameters of Feige 48. The periods of Feige 48 correspond to low-order modes with adjacent values of $k$ and with $\ell=0,1$, and 2 . The relative dispersion between the observed and computed periods for the best model approaches a mere $\sim 0.005 \%$ on average, i.e., close to the actual accuracy of the observations. The structural parameters of Feige 48 obtained from that study are $T_{\text {eff }}=29580 \mathrm{~K} \pm 370 \mathrm{~K}$ (fixed to its spectroscopic value), $\log g=5.437 \pm 0.006, \log M_{\text {env }} / M_{*}=-2.97 \pm 0.09$, and $M_{*}=0.460 \pm 0.008 M_{\odot}$. Interpreting a posteriori the presence of multiplet components as an effect of solid-body rotation, a rotation period of $P=\overline{1-C_{k l}} / \overline{\Delta v}=9.44 \pm 1.18 \mathrm{~h}$ was derived. Within the quoted uncertainies, this is compatible with the idea of spin-orbit synchronism in the system. Furthermore, this value of the spin period leads to an estimate of the equatorial velocity of $V_{\text {eq }}=27.6 \pm 3.9 \mathrm{~km} \mathrm{~s}^{-1}$. With the limit of $V \sin i \lesssim 5 \mathrm{~km} \mathrm{~s}^{-1}$ fixed by Heber et al. (2000), this suggests an inclination angle $i \lesssim 10.4 \pm 1.7^{\circ}$. 
Following these results and recent improvements in our diagnostic tools for asteroseismology, we felt that it would be potentially rewarding to test the robustness of these findings by treating rotation at the outset and performing again the seismic analysis on the basis, this time, of all available observed periods. We note that a similar approach will be necessary to analyze rapidly pulsating $\mathrm{sdB}$ stars in which rotation plays an obvious role, such as PG 1605+072 (Heber et al. 1999) or KPD 1930+2752 (Billères et al. 2000).

\section{New asteroseismic analysis}

\subsection{Numerical Tools}

The method developed to perform objective asteroseismic studies of sdB pulsators has been described in some detail in Charpinet et al. (2005b). It implements the so-called forward modeling method, which consists of comparing quantitatively computed pulsation periods for large batches of stellar models to the observed periods of the star under interest. This approach is built on the specific requirement of global optimization, i.e., pulsation spectra computed from sdB models must match all the observed periods simultaneously. For the present analysis, we used the "package" developed in the past few years by the Toulouse group (see again Charpinet et al. 2005b), modified to incorporate the effect of rotational splitting. The seismic analysis follows a multi-step procedure. The first step consists of calculating the internal structure of the subdwarf B star given four fundamental parameters: the effective temperature $T_{\text {eff }}$, the surface gravity $\log g$, the total mass of the star $M_{*}$, and the logarithmic fractional mass of the hydrogen-rich envelope $\log q(\mathrm{H}) \equiv \log \left[M(\mathrm{H}) / M_{*}\right]$. These are the natural parameters needed to fully define the socalled "second generation" models appropriate for sdB asteroseismology. We recall that these models are static structures expanding as deep as $\log q \equiv \log \left(1-M(r) / M_{*}\right) \simeq-0.05$ that incorporate nonuniform abundance profiles of iron derived from detailed microscopic diffusion calculations assuming an equilibrium between gravitational settling and radiative levitation, a key ingredient to construct reliable models of pulsating sdB stars (see, e.g., Fontaine et al. 2006b). In a second step, we compute the adiabatic pulsation properties of the model using an efficient and robust code based on finite element techniques (see Brassard et al. 1992). Next, for each eigenfrequency, the associated multiplets assuming a spherically symmetric internal rotation law $\Omega(r)$ (we do not consider axisymmetric differential rotation here) are calculated with the perturbative method to first order:

$$
\begin{aligned}
\delta \omega_{k l m} & =\omega_{k l m}-\omega_{k l} \\
& =-m \frac{\int_{0}^{R} \Omega(r)\left(\xi_{r}^{2}+L^{2} \xi_{h}^{2}-2 \xi_{r} \xi_{h}-\xi_{h}^{2}\right) \rho r^{2} \mathrm{~d} r}{\int_{0}^{R}\left(\xi_{r}^{2}+L^{2} \xi_{h}^{2}\right) \rho r^{2} \mathrm{~d} r}
\end{aligned}
$$

where the unperturbed eigenfunctions $\xi_{r}$ and $\xi_{h}$ are given by the adiabatic pulsation code, and $L^{2} \equiv \ell(\ell+1)$. The frequencies produced this way, each mode now being identified by its $k, \ell$ and $m$ indices, are then employed in a double-optimization procedure aimed at determining the optimal solutions that minimize a merit function defined as

$S^{2}=\sum_{i=1}^{N_{\mathrm{obs}}}\left(P_{\mathrm{obs}}^{i}-P_{\mathrm{th}}^{i}\right)^{2}$

where $N_{\text {obs }}$ is the number of observed periodicities, nine in the case of Feige 48. The first optimization finds, for a given equilibrium model, the mode identification leading to the best possible simultaneous match of all the observed periods to the computed periods. The latter, $P_{\text {th }}^{i}$, are found among the available period spectrum $\left(2 \pi / \omega_{k l m}\right)$ computed for that model. Then, the optimal model solutions are searched by finding minima of the quantity $S^{2}\left(T_{\text {eff }}, \log g, \log q(\mathrm{H}), M_{*}, P_{\text {rot }}\right)$ with a dedicated optimization code based on a Genetic Algorithm (GA) designed to explore the vast model parameter space. With this method, the mode identification (here the indices $k, \ell, m$ ) for each observed period is obtained as a byproduct of the optimization procedure. We point out that this mode identification can be, in principle, partially tested by independent techniques such as multicolor photometry or time resolved spectroscopy.

The introduction of the effects of stellar rotation on pulsations avoids us the need for making a priori identifications of the central $m=0$ component of an observed multiplet. Hence, quite importantly, all observed periods can now be used for the asteroseismic analysis in an objective way. At this stage, to make it perfectly clear, we note that the equilibrium models that we use are still spherical (non-rotating) structures, but we do introduce rotational splitting at the level of first-order perturbation.

\subsection{Search for the optimal model assuming solid-body rotation}

Among the rotation laws implemented in our codes, our first experiment was to assume that Feige 48 is a solid-body rotator (i.e., $\Omega(r)=\Omega=$ constant). In that case, only one parameter is needed to characterize the rotation of the star, and that is $P_{\text {rot }}=2 \pi / \Omega$, the rotation period of the star. Following the previous work of Charpinet et al. (2005a), the effective temperature derived from the spectroscopic measurement, $T_{\text {eff }}=29580 \mathrm{~K}$, is adopted as the best estimate of this parameter. This is because this parameter is much more accurately determined from spectroscopy (given the weak temperature dependence of the pulsation periods, see below). Thus, the optimization is performed in a four-dimensional parameter space defined by the remaining free parameters $\log g, \log q(\mathrm{H}), M_{*}$, and $P_{\text {rot }}$. Initial boundaries of the search domain were defined as follows: $5.35 \leq \log g \leq 5.60$, $-5.20 \leq \log q(\mathrm{H}) \leq-2.00$, and $0.30 \leq M_{*} / M_{\odot} \leq 0.70$ for the structural parameters. The range for $P_{\text {rot }}$ is taken from $20000 \mathrm{~s}$ to $60000 \mathrm{~s}$, thus bracketing the rough period determination obtained by taking the inverse of the mean frequency spacing seen in the data $(1 /[28 \mu \mathrm{Hz}] \sim 35700 \mathrm{~s})$. The limits on the surface gravity are loosely set according to the spectroscopic estimates, whereas the ranges for $\log q(\mathrm{H})$ and $M_{*}$ rely on considerations derived from stellar evolution constraints including various possible formation scenarios (see Han et al. 2002, 2003). All modes of degree $\ell=0$ to 2 are considered. This upper limit for the degree $\ell$ corresponds to the minimum value that can account for the mode density in the observed period range. The presence of modes with higher $\ell$ values cannot formally be ruled out, but our approach explicitly excludes this possibility. We stress however that, in the specific case of Feige 48, modes of degree $\ell \leq 2$ can fully explain the observed pulsation spectrum, as shown below.

Within the search domain specified, the optimization code identified several families of models that present potentially good matches of the observed periods. Most of them, however, can be discarded upon closer inspection, either on the basis of obvious inconsistency with spectroscopy in terms of surface gravity, or because the inferred multiplets in the mode identification do not reproduce satisfactorily the nearly equal frequency spacings seen in the pulsation spectrum of Feige 48. These unsatisfactory solutions filtered out, only one of the best-fit models turns out to be fully consistent with the spectroscopic estimates 

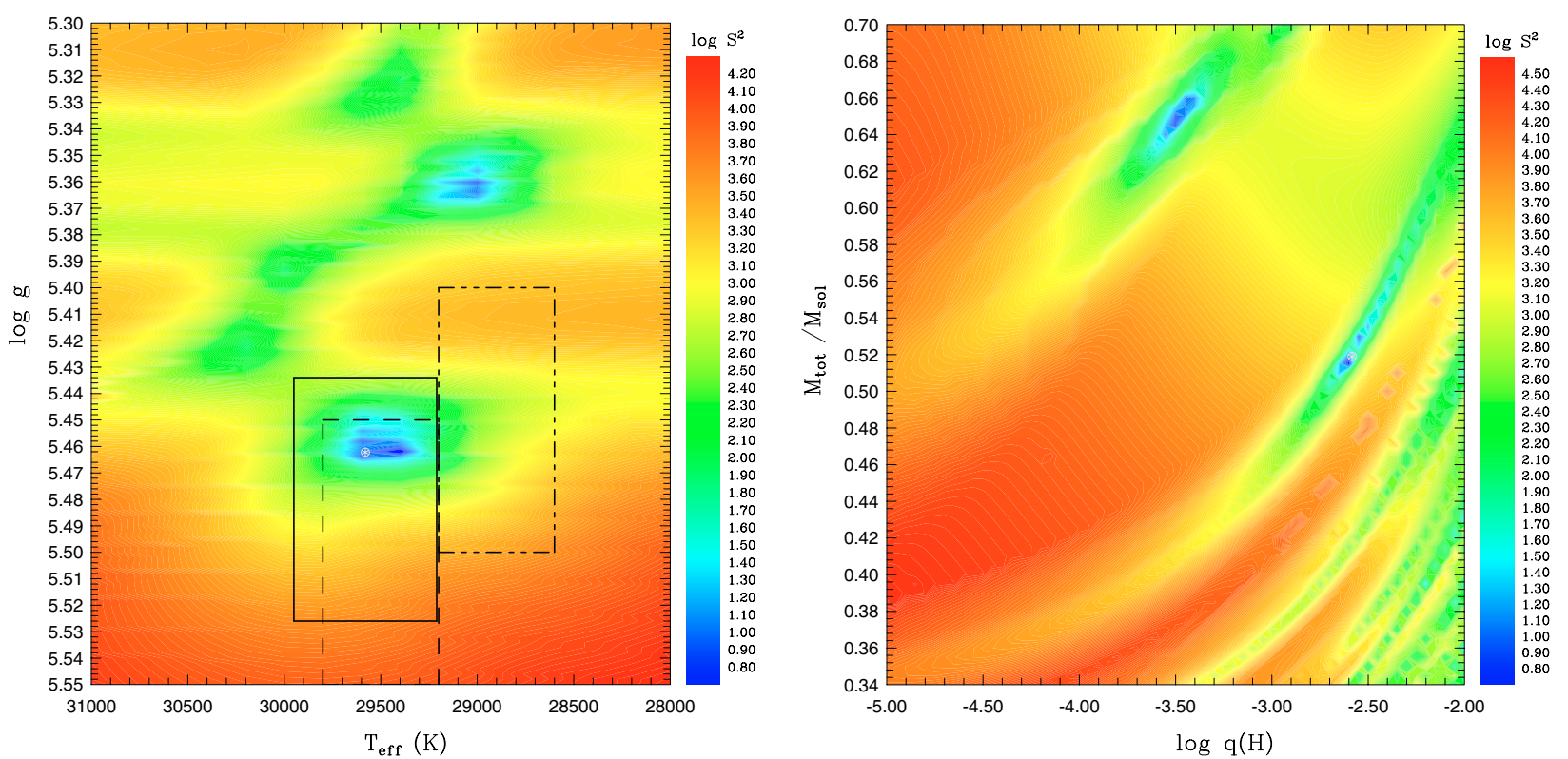

Fig. 1. Left panel: slice of the $S^{2}$ function (in $\log$ units) along the $\log g-T_{\text {eff }}$ plane at fixed parameters $M_{*}$ and $\log q(\mathrm{H})$ set to their optimal values. The solid-line rectangle materializes our spectroscopic estimate (Charpinet et al. 2005a) with its uncertainties for the atmospheric parameters of Feige 48, while the dashed-line and dot-dashed-line rectangles represent the Heber et al. (2000) and Koen et al. (1998) spectroscopic measurements, respectively. Right panel: slice of the $S^{2}$ function (in $\log$ units) along the $M_{*}-\log q(\mathrm{H})$ plane at fixed parameters $\log g$ and $T_{\text {eff }}$ set to their optimal values. In both panels, the best-fit model is indicated by a yellow mark, and the rotation period is fixed to $32500 \mathrm{~s}$ with the hypothesis of a solid-body rotator.

of $\log g$ while offering a meaningful identification of multiplets relative to the observed frequency spacings. This preferred solution is found at $\log g=5.462, \log q(\mathrm{H})=-2.58, M_{*}=0.519 M_{\odot}$ and $P_{\text {rot }}=32500 \mathrm{~s}$. With a $S^{2}$ value of 0.59 , this model provides an excellent simultaneous match to the nine periods observed in the pulsating sdB star Feige 48 (see below).

The maps shown in Fig. 1 illustrate the behavior of the $S^{2}$ function in the vicinity of our preferred solution (see figure caption for details), with a star rotation fixed to its optimal value of $32500 \mathrm{~s}$ in both panels. These maps show slices of the $S^{2}$ function, respectively along the $\log g-T_{\text {eff }}$ plane (with the mass and envelope thickness fixed to their optimal values) for the left panel, and along the $M_{*}-\log q(\mathrm{H})$ plane (with the effective temperature and the surface gravity fixed to their optimal values) for the right panel. Best fitting models (corresponding to low values of $S^{2}$ ) appear as blue regions, while red areas represent comparatively bad fits. Considering the logarithmic scale used to represent the merit function on these plots, we stress that the blue regions correspond to very well-defined minima. The left panel shows two deep "pits", one centered around $\log g \sim 5.46$ and the other located near $\log g \sim 5.36$. The last one is in obvious conflict with all spectroscopic estimates of $\log g$ and was indeed immediately rejected on this basis. In the right panel, one elongated oblique "valley" is centered around the solution at $\sim 0.52 M_{\odot}$ and $\log q(\mathrm{H}) \sim-2.5$. A second blue valley indicates the presence of another potential family of solutions at very high mass $\left(\sim 0.65 M_{\odot}\right)$. However, a closer inspection of this model has revealed that the inferred mode identification does not match the nearly regular frequency spacings observed in Feige 48. In this case, the optimal mode identification, although not bad at the strict level of average dispersion between the observed and computed periods, produce modeled multiplets resulting in very irregular frequency spacings, at odds with the clear rotational splitting patterns observed in this star. To account for these, one would have to invoke strong higher-order perturbative effects caused by rotation, which are highly improbable for a moderate rotator like Feige $48^{1}$. Hence, on that basis, this highmass model can be confidently rejected.

A more subtle type of degeneracy of the asteroseismic solution among the chosen family of model exists. These degeneracies appear when a change in one of the model parameters can be almost exactly compensated by a change in another parameter, leaving the computed periods practically unchanged. Such degeneracies were encountered in all studies of EC 14026 pulsators led so far, and occur in a similar way in the present analysis of Feige 48. They were already noted and described at length in the first asteroseismic analysis without rotation of Feige 48 (Charpinet et al. 2005a). First, there is a correlation between the thickness of the envelope $\log q(\mathrm{H})$ and the effective temperature $T_{\text {eff }}$ : a change of $\log q(\mathrm{H})$ while keeping the parameter $M_{*}$ fixed to its optimal value produces a shift of the position of the local minimum, essentially along the temperature axis. This trend is illustrated in the map shown in Fig. 2, which represents the projection of the $\log q(\mathrm{H})$ axis onto the $\log g-T_{\text {eff }}$ plane. More precisely, the logarithm of the $S^{2}$ value associated with each grid point shown on the map is the minimum value found among all the values of the merit function obtained at the specific grid point of $T_{\text {eff }}$ and $\log g$, and with the stellar mass $M_{*}$ set to its optimal value $\left(0.519 M_{\odot}\right)$, but with the parameter $\log q(\mathrm{H})$ varying between -5.20 and -2.00 . The labelled axis positioned along the valley associated with the preferred solution (at $\log g \sim 5.46$ ) indicates the position of the local minimum of the merit function $S^{2}$ as a function of $\log q(\mathrm{H})$ near the optimal solution. There is a clear monotonic trend showing that the minimum shifts from higher to lower $T_{\text {eff }}$ as the envelope mass of the star increases. Again, note that spectroscopy is essential

\footnotetext{
${ }^{1}$ Indeed, we think that second-order perturbative effects due to rotation already manifest themselves in the non-strictly regular frequency spacing observed within a given multiplet, as observed in Table 1 . Such small variations would be consistent with expectations for the magnitude of such higher-order effects in a star rotating like Feige 48.
} 


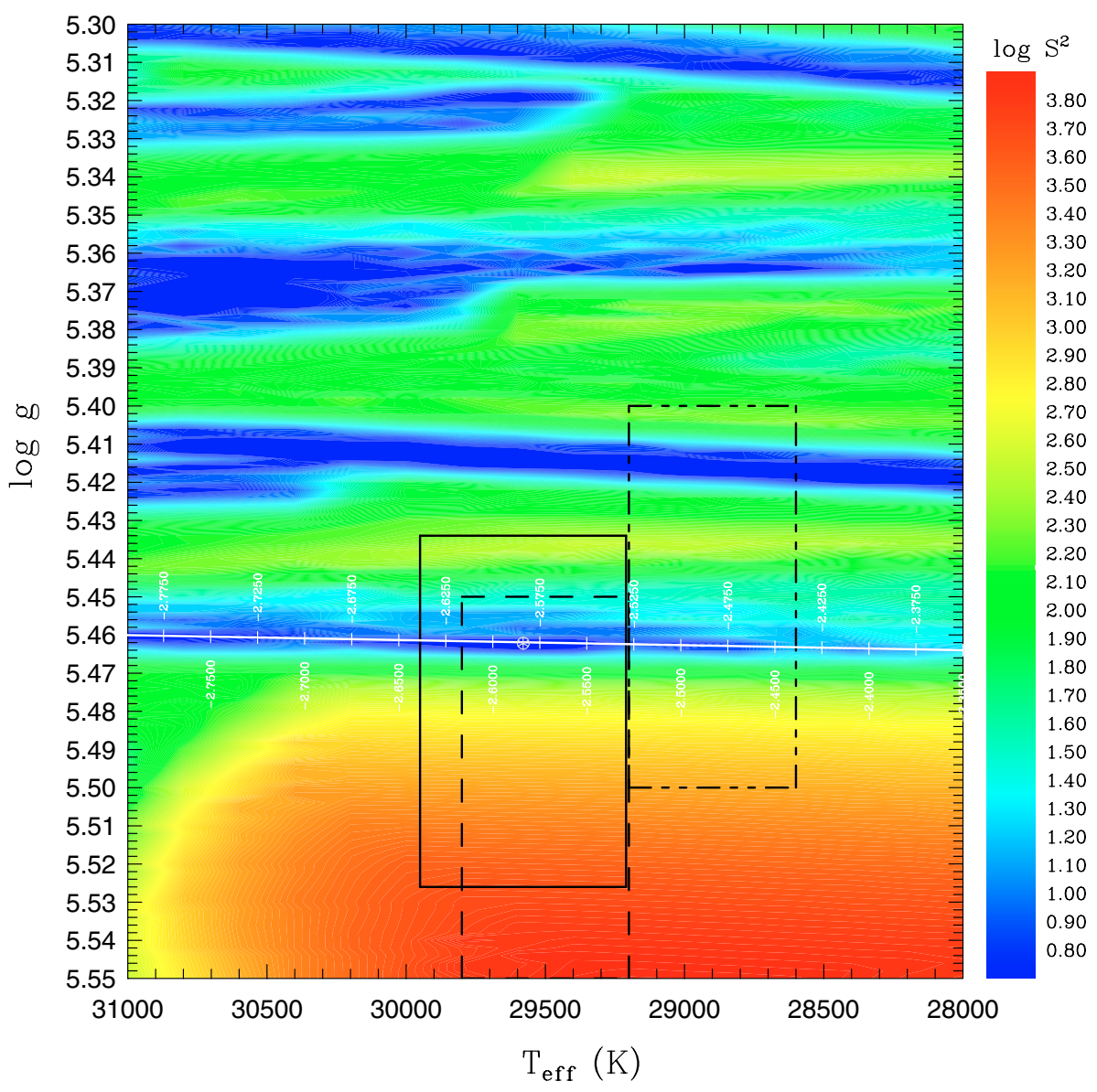

Fig. 2. Slice of the "projected" $S^{2}$ function (in log units) along the $\log g-T_{\text {eff }}$ plane at fixed parameter $M_{*}$, set to its optimal value from the best-fit model solution $\left(M_{*}=0.519 M_{\odot}\right)$. The rotation period is fixed to $32500 \mathrm{~s}$, with the hypothesis of a solid-body rotator. The projected log $q(\mathrm{H})$ parameter was varied between -2.00 and -5.20 , in steps of 0.025 . The labelled axis positioned along the valley of minimum $S^{2}$ for the given values of $\log q(\mathrm{H})$. The solid-line, dashed-line and dot-dashed line rectangles represent, with their uncertainties, the spectroscopic estimates for the atmospheric parameters of Feige 48 from Charpinet et al. (2005a), Heber et al. (2000) and Koen et al. (1998), respectively.

to choose among the valleys regularly repeated at lower surface gravities. These are linked with the duplicities of the asteroseismic solution caused by the "mode jumping" phenomenon, i.e., models with the same $(\ell, m)$ identification, but with a shift of $\Delta k=1$ (see Randall et al. 2007). Such valleys also occur when we explore changes applied to the total mass of the star. This time, Fig. 3 represents the projection of the $M_{*}$-axis onto the $\log g-T_{\text {eff }}$ plane. The parameter $\log q(\mathrm{H})$ was set to its optimal value, and the total mass was varied between 0.30 and $0.70 M_{\odot}$. The map clearly shows that a correlation exists between the parameters $M_{*}, T_{\text {eff }}$ and, to a much lesser extent, $\log g$. A change in $M_{*}$ generates a shift in $T_{\text {eff }}$ (and slightly in $\log g$ ) of the position of the $S^{2}$ minimum, as indicated by the labelled axis along the region of best-fit models. This leads to line-degeneracies, clearly apparent in Fig. 3 as long and flat valleys of minimum $S^{2}$. Again, the additional constraints brought by spectroscopy are crucial, not only for choosing the right valley along the $\log g$ axis, but also for selecting the appropriate section along the line of degeneracy which corresponds to the "correct" solution. This kind of degeneracy justifies a posteriori the strategy of setting the $T_{\text {eff }}$ value according to spectroscopic estimates for the search of the optimal models with the GA-code.

Finally, the optimal value for the rotation period of Feige 48 obtained under the assumption of solid-body rotation is of utmost interest here. Indeed, our inferred value for the spin period of the sdB component, $P_{\text {rot }}=32500$ s (i.e., $9.028 \mathrm{~h}$ ), is remarkably consistent with the orbital period of the system, $P_{\text {orb }}$ $=9.024 \pm 0.072 \mathrm{~h}$, as determined from the radial velocity measurements of O'Toole et al. (2004). In this context, Fig. 4 illustrates the behavior of the merit function $S^{2}$ as a function of the rotation period (keeping all other parameters fixed at their optimal values). There exists a very well defined minimum corresponding, indeed, to the orbital period of the system. From the shape of the $S^{2}$ curve, and assuming quite conservatively that all the uncertainty in $S^{2}$ is due exclusively to a variation in $P_{\text {rot }}$ alone, we have evaluated a generous $1-\sigma$ uncertainty of $\sim 0.48 \mathrm{~h}$ for the rotation period. Hence, unless a very improbable accidental coincidence between the inferred values of $P_{\text {rot }}$ and $P_{\text {orb }}$ occurs in this star, this result strongly suggests that the Feige 48 binary system is tidally locked.

\subsection{Period fit and mode identification}

Details on the period fit and mode identification resulting from the optimal model isolated in the previous subsection are given in Table 2. The identification (or $\ell, k, m$ indices of each observed period) is given, as well as the relative difference $\Delta P / P$ for each pair $\left(P_{\mathrm{obs}}, P_{\mathrm{th}}\right)$ of associated modes. Table 2 also provides a comparison between calculated (according to Eq. (1)) and observed frequency spacings. On average, the relative dispersion of the period fit is $\overline{\Delta P / P} \sim 0.06 \%$ (or $\overline{\Delta P}=0.22 \mathrm{~s}$ on an absolute scale). It is inferior to the best model from the first analysis, but 


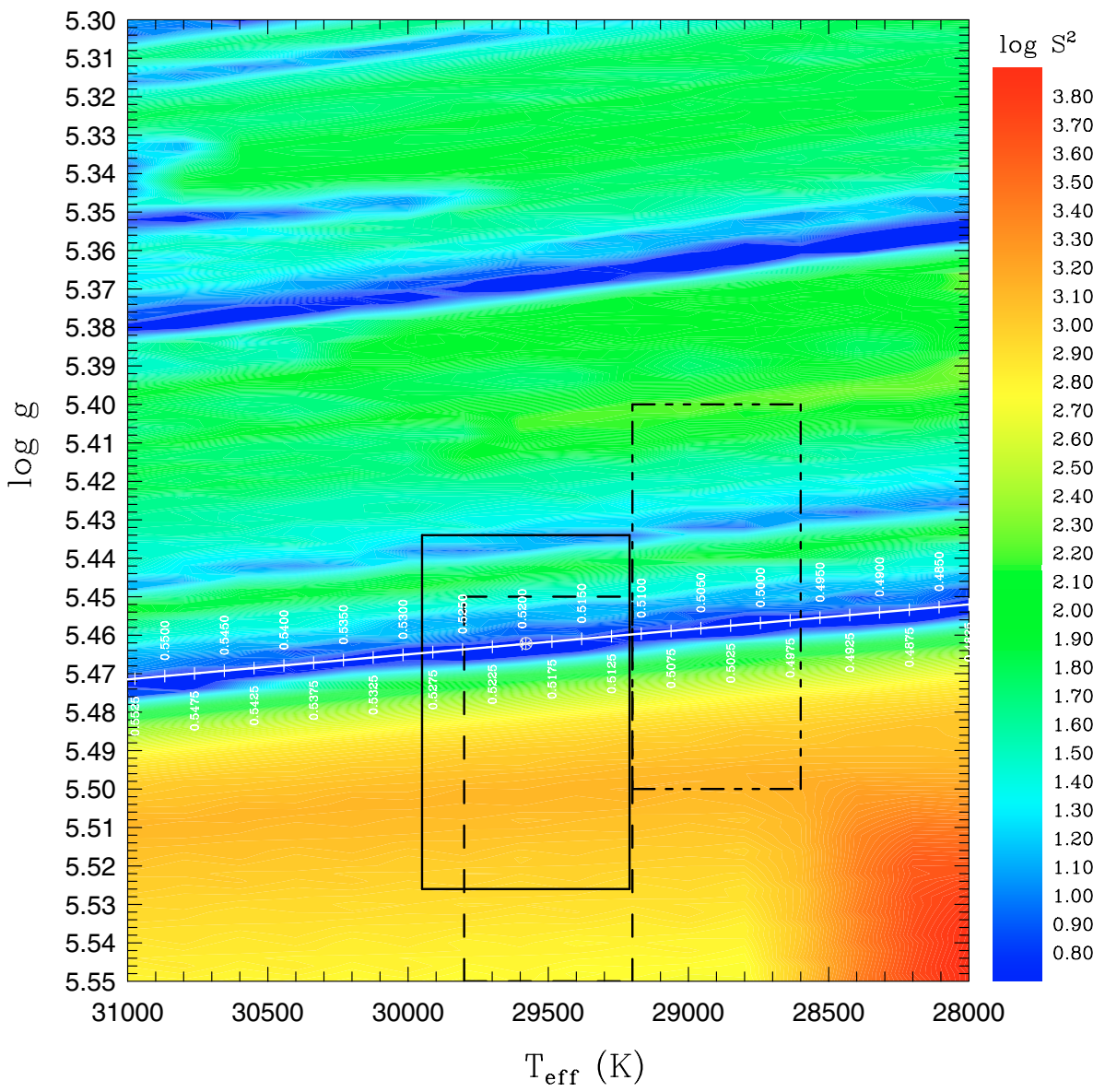

Fig. 3. Slice of the "projected" $S^{2}$ function (in $\log$ units) along the $\log g-T_{\text {eff }}$ plane at fixed parameter $\log q(\mathrm{H})$, set to its optimal value from the best-fit model solution $(\log q(\mathrm{H})=-2.52)$. The rotation period is fixed to $32500 \mathrm{~s}$, with the hypothesis of a solid-body rotator. The projected $M_{*}$ parameter was varied between $0.40 M_{\odot}$ and $0.60 M_{\odot}$, in steps of $0.0025 M_{\odot}$. The labelled axis positioned along the valley of minimum $S^{2}$ for the given values of $M_{*}$. The solid-line, dashed-line and dot-dashed line rectangles represent, with their uncertainties, the spectroscopic estimates for the atmospheric parameters of Feige 48 from Charpinet et al. (2005a), Heber et al. (2000) and Koen et al. (1998), respectively.

the latter was based on the simultaneous fit of four periods only, instead of nine in the present analysis. Hence this overall degradation of the best-fit solution was to be expected. We point out on that matter that the present model still provides an excellent fit of the periods by current standards in sdB asteroseismology.

Looking into the details of the mode identification, we note that the period $f_{3}$ is identified with the fundamental radial mode, as in the former analysis of Charpinet et al. (2005a). This eliminates the possibility alluded to above that it could have been the $f_{1}^{+}$component of the $f_{1}$ multiplet. The dominant (in amplitude) period $f_{2}$ and its group are found to be the three $m$ members of the $l=1, k=1 p$-mode. The doublet $f_{1}$ is the $l=2 f$-mode, with the identification $m=+1$ and +2 for its two components. A similar behavior is observed in the $f_{4}$ group, identified with the $\ell=2, k=1 g$-mode, with $m=0,+1$ and +2 for its components. Quite interestingly, the $(k, \ell)$ identification remain the same as in the optimal model proposed by Charpinet et al. (2005a), but the central components $(m=0)$ of the $f_{1}$ and $f_{4}$ multiplets are different. We come back to that point below.

In the absence of a practical nonlinear theory, it is not possible to explain here why modes with positive values of $m$ would show higher amplitudes in the $f_{1}$ and $f_{4}$ multiplets. This is particularly puzzling in view of the fact that the Feige 48 system is believed to be seen at low inclination, a geometry unfavorable a priori for the detection of $|m|=2$ modes. Perhaps, as a speculation, the binary nature of the system has an influence on the

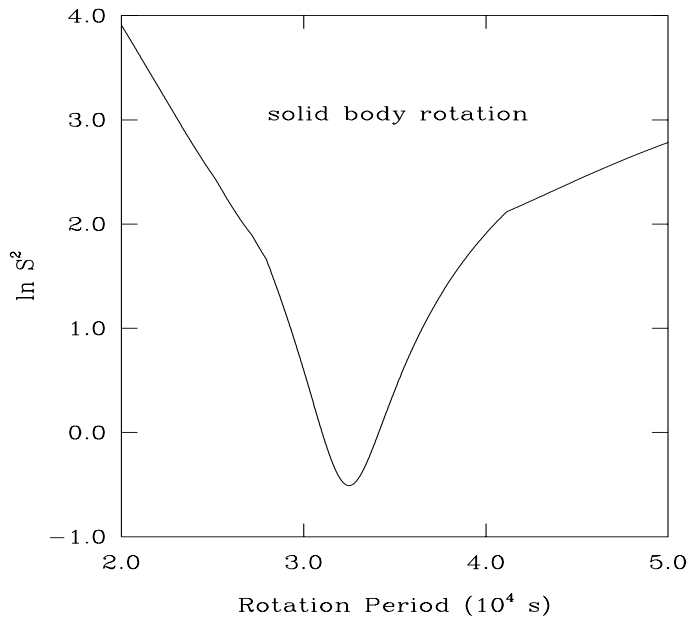

Fig. 4. Merit function $S^{2}$ (in logarithmic units) versus rotation period (in seconds) in the hypothesis of a solid-body rotator. All other structural parameters are fixed to their optimal values.

stability of sectorial modes with $l=2$ via the tidal forces. It remains to be seen if time-resolved spectrocopy of Feige 48 could confirm (or infirm) the $m$ identifications proposed here. 
Table 2. Period fit and derived mode identification for the optimal model of Feige 48.

\begin{tabular}{ccrcccccc}
\hline \hline$\ell$ & $k$ & \multicolumn{1}{c}{$\begin{array}{c}P_{\text {obs }} \\
(\mathrm{s})\end{array}$} & $\begin{array}{c}P_{\text {th }} \\
(\mathrm{s})\end{array}$ & $\begin{array}{c}\Delta P / P \\
(\%)\end{array}$ & $\begin{array}{c}\Delta f_{\text {calc }} \\
(\mu \mathrm{Hz})\end{array}$ & $\begin{array}{c}\Delta f_{\text {obs }} \\
(\mu \mathrm{Hz})\end{array}$ & Comments \\
\hline 0 & 2 & 0 & $\ldots$ & 237.539 & $\ldots$ & $\ldots$ & $\ldots$ & \\
0 & 1 & 0 & $\ldots$ & 292.924 & $\ldots$ & $\ldots$ & $\ldots$ & $f_{3}$ singlet \\
0 & 0 & 0 & 352.450 & 352.072 & 0.107 & $\ldots$ & $\ldots$ & \\
1 & 2 & -1 & $\ldots$ & 288.066 & $\ldots$ & 30.36 & $\ldots$ & \\
1 & 2 & 0 & $\ldots$ & 290.608 & $\ldots$ & 30.36 & $\ldots$ & \\
1 & 2 & +1 & $\ldots$ & 293.194 & $\ldots$ & 30.36 & $\ldots$ & \\
1 & 1 & -1 & 344.068 & 344.116 & -0.0141 & 28.62 & 28.90 & \multirow{2}{*}{ triplet } \\
1 & 1 & 0 & 347.530 & 347.540 & -0.0028 & 28.62 & $\ldots$ & \\
1 & 1 & +1 & 350.746 & 351.032 & -0.0815 & 28.62 & 26.40 & \\
2 & 1 & -2 & $\ldots$ & 280.775 & $\ldots$ & 30.59 & $\ldots$ & \\
2 & 1 & -1 & $\ldots$ & 283.208 & $\ldots$ & 30.59 & $\ldots$ & \\
2 & 1 & 0 & $\ldots$ & 285.683 & $\ldots$ & 30.59 & $\ldots$ & \\
2 & 1 & +1 & $\ldots$ & 288.202 & $\ldots$ & 30.59 & $\ldots$ & \\
2 & 1 & +2 & $\ldots$ & 290.766 & $\ldots$ & 30.59 & $\ldots$ & \\
2 & 0 & -2 & $\ldots$ & 332.382 & $\ldots$ & 30.32 & $\ldots$ & \\
2 & 0 & -1 & $\ldots$ & 335.766 & $\ldots$ & 30.32 & $\ldots$ & \\
2 & 0 & 0 & $\ldots$ & 339.219 & $\ldots$ & 30.32 & $\ldots$ & \\
2 & 0 & +1 & 343.027 & 342.744 & +0.0824 & 30.32 & 25.02 & $f_{1}$ doublet \\
2 & 0 & +2 & 345.997 & 346.343 & -0.1001 & 30.32 & $\ldots$ & \\
2 & 1 & -2 & $\ldots$ & 366.704 & $\ldots$ & 28.40 & $\ldots$ & \\
2 & 1 & -1 & $\ldots$ & 370.563 & $\ldots$ & 28.40 & $\ldots$ & \\
2 & 1 & 0 & 374.280 & 374.504 & -0.0598 & 28.40 & 29.5 & \\
2 & 1 & +1 & 378.461 & 378.529 & -0.0181 & 28.40 & $\ldots$ & $f_{4}$ triplet, $g$-mode \\
2 & 1 & +2 & 382.988 & 382.643 & +0.0902 & 28.40 & 31.2 & \\
\hline & & & & & & & & \\
& & & & $\ldots$ & & \\
\end{tabular}

\subsection{Hypothesis of differential core rotation}

The idea of differential core rotation (and, in particular, rapidly rotating core remnants) for $\mathrm{sdB}$ stars was proposed recently by Kawaler \& Hostler (2005). These authors suggested that $\mathrm{sdB}$ stars in general should retain fast rotating cores as a relic of their past evolution on the RGB. Since differential rotation, with a high-enough contrast, can leave a signature on the period spectrum of a pulsating star, we found it interesting to investigate this possibility on the basis of the nine periods observed in Feige 48. Following the properties of the models investigated in Kawaler \& Hostler (2005), we constructed a differential rotation law characterized by two regions (the core and the envelope) rotating each as solid structures, with a sheer layer between the core and the envelope located at $0.3 R_{*}$. With this prescription, the rotation profile of the star closely mimics the rotation structure of the Kawaler \& Hostler (2005) models. In our experiment, the envelope rotation period was fixed at the optimal value of $32500 \mathrm{~s}$ found for solid-body rotation, and the core rotation was varied from a period of $4500 \mathrm{~s}$ to $40000 \mathrm{~s}$, usually in steps of $1000 \mathrm{~s}$. The merit function $S^{2}$ was then minimized over the remaining free model parameters for each fixed core period. The result is shown in Fig. 5, which we find exceptionally suggestive.

From that figure, a very fast core rotation can immediately be rejected as it leads to much poorer values of the merit function (note the logarithmic scale used for the ordinate axis in Fig. 5). Fast core rotation produces a period spectrum increasingly deviant from the observed period spectrum of Feige 48. We find that the best $S^{2}$ is obtained for a core rotation period of $\sim 30000 \mathrm{~s}$, which, taken at face value, would suggest a slight differential rotation for Feige 48. However, from a statistical point of view, this model with $S^{2}=0.42$ is not significantly different, within the 1- $\sigma$ uncertainties, from the solution corresponding to pure solid-body rotation having $S^{2}=0.59$. It is clear, however, that the hypothesis of a fast rotating core such

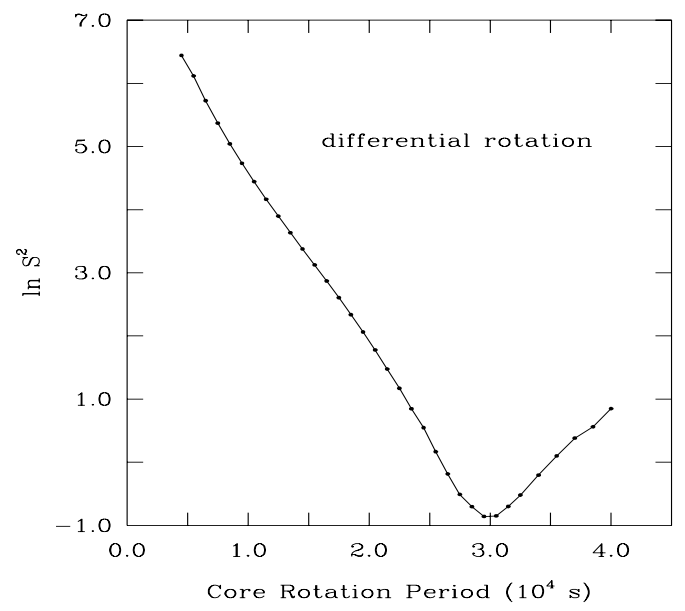

Fig. 5. Merit function $S^{2}$ (in logarithmic units) as a function of the rotation period of the core (in s). The surface rotation is fixed at the optimal value of $32500 \mathrm{~s}$ found for solid-body rotation.

as that envisioned by Kawaler \& Hostler (2005) must be completely excluded for Feige 48. Moreover, we also find that a core rotating more slowly than the envelope is not an option, as the merit function $S^{2}$ increases again for longer core rotation periods. This behavior results again in a very well defined minimum in the $S^{2}$ function. Hence, taking into account the uncertainties, Fig. 5 provides a very strong indication that Feige 48 rotates as a solid body from its surface down to its center. This is the test that we were seeking.

\section{Comparison with previous work}

The first attempt to match the periods observed in Feige 48 was realized by Reed et al. (2004), using standard evolutionary models of the extreme horizontal branch with uniform solar 
Table 3. Comparison of merit functions, structural parameters, rotation period, and mode identification for three models with the same $(k, \ell)$ values, but different $m$ values (see text for details).

\begin{tabular}{|c|c|c|c|c|c|c|c|c|c|c|}
\hline ID & $P_{\mathrm{obs}}(\mathrm{s})$ & $\overline{\ell \ell}$ & $k$ & $m$ & $\overline{\ell \ell}$ & $k$ & $m$ & $\ell$ & $k$ & $m$ \\
\hline & 343.027 & 2 & 0 & +1 & 2 & 0 & 0 & 2 & $\overline{0}$ & +1 \\
\hline$f_{1}$ doublet & 345.997 & 2 & 0 & +2 & 2 & 0 & +1 & 2 & 0 & 0 \\
\hline \multirow{3}{*}{$f_{2}$ triplet } & 344.068 & 1 & 1 & -1 & 1 & 1 & -1 & 1 & 1 & -1 \\
\hline & 347.530 & 1 & 1 & 0 & 1 & 1 & 0 & 1 & 1 & 0 \\
\hline & 350.746 & 1 & 1 & +1 & 1 & 1 & +1 & 1 & 1 & +1 \\
\hline$f_{3}$ & 352.450 & 0 & 0 & 0 & 0 & 0 & 0 & 0 & 0 & 0 \\
\hline \multirow{3}{*}{$f_{4}$ triplet } & 374.280 & 2 & 1 & 0 & 2 & 1 & 0 & 2 & 1 & -1 \\
\hline & 378.461 & 2 & 1 & +1 & 2 & 1 & +1 & 2 & 1 & 0 \\
\hline & 382.988 & 2 & 1 & +2 & 2 & 1 & +2 & 2 & 1 & +1 \\
\hline \multicolumn{2}{|c|}{ Merit function } & \multicolumn{3}{|c|}{$S^{2} \simeq 0.6$} & \multicolumn{3}{|c|}{$S^{2} \simeq 0.9$} & \multicolumn{3}{|c|}{$S^{2} \simeq 2.6$} \\
\hline \multicolumn{2}{|c|}{$\log g$} & \multicolumn{3}{|c|}{5.462} & \multicolumn{3}{|c|}{5.450} & \multicolumn{3}{|c|}{5.437} \\
\hline \multicolumn{2}{|l|}{$M_{*} / M_{\odot}$} & \multicolumn{3}{|c|}{0.519} & \multicolumn{3}{|c|}{0.488} & \multicolumn{3}{|c|}{0.460} \\
\hline \multicolumn{2}{|c|}{$\log \left(M_{\mathrm{env}} / M_{\odot}\right)$} & \multicolumn{3}{|c|}{-2.52} & \multicolumn{3}{|c|}{-2.72} & \multicolumn{3}{|c|}{-2.97} \\
\hline \multicolumn{2}{|c|}{ Rotation period } & \multicolumn{3}{|c|}{$P_{\mathrm{rot}} \simeq 32500 \mathrm{~s}$} & \multicolumn{3}{|c|}{$P_{\mathrm{rot}} \simeq 30500 \mathrm{~s}$} & \multicolumn{3}{|c|}{$P_{\mathrm{rot}} \simeq 29500$} \\
\hline
\end{tabular}

$(Z=0.02)$ metallicity. On the basis of the five periods determined from their campaign (see last column of Table 1), they proposed a model with structural parameters of $T_{\text {eff }}=29635 \mathrm{~K}$, $\log g=5.518, M_{\mathrm{env}}=0.0025 M_{\odot}$ and $M_{*}=0.4725 M_{\odot}$. However, this model suffers from a serious inconsistency, in the sense that it failed to match simultaneously the five periods observed. This concerns particularly the $378.5 \mathrm{~s}$ period, which is not satisfactorily reproduced by this model even when invoking high $\ell$ degree modes. As demonstrated by Charpinet et al. (2005a), this situation is due to the absence, in their standard models, of a key ingredient : microscopic diffusion processes that produce nonuniform chemical profiles (especially for iron) in the envelope of these stars, which must be taken into account for accurate quantitative asteroseismological studies of sdB stars.

It is more instructive to compare our results with those of Charpinet et al. (2005a). As indicated above, the $(k, \ell)$ identification obtained in our optimal model is the same as that found by these authors in their best-fit model. However, there are significant differences - 10 to $15 \%$ - between the inferred structural properties of Feige 48 in the two studies. These differences can be traced back to the fact that our assigned $m=0$ components of the $f_{1}$ and $f_{4}$ multiplets differ in period by, respectively, $\sim 6.8 \mathrm{~s}$ and $\sim 4.2 \mathrm{~s}$ from the assumed central components in that previous study. Such period differences are sufficiently large to lead to different optimal physical models.

In a numerical experiment, we redid our asteroseismic analysis using all nine available periods, but we imposed at the outset the $m=0$ identifications assumed by Charpinet et al. (2005a). Specifically, as was done in that paper, we assigned a priori the value $m=0$ to the $f_{1}, f_{2}, f_{3}$, and $f_{4}$ modes listed in Table 1 . The difference with the investigation of Charpinet et al. (2005a) is that the search for the optimal model was carried out in a 4D instead of a space with the addition of $P_{\text {rot }}$ to the other three usual parameters $\left(\log g, \log q(\mathrm{H})\right.$, and $\left.M_{*}\right)$ after having fixed the effective temperature to its spectroscopic value. Moreover, the aim of our new search was to fit simultaneously all nine observed periods as opposed to only the four assumed $m=0$ components as done in Charpinet et al. (2005a).

The results of this additional exercise are summarized in the RHS column of Table 3. For comparison, our previous results are reported in the LHS column of the table. Not surprisingly, we recover exactly the same four primary parameters found by Charpinet et al. (2005a), i.e., $T_{\text {eff }}=29580 \mathrm{~K}, \log g=5.437$, $M_{*}=0.460 M_{\odot}$, and $\log \left(M_{\text {env }} / M_{\odot}\right)=-2.97$. However, by fitting an extra five periods in the present case, the global match has significantly worsened and we now find a merit function $S^{2} \simeq 2$.6. This is about four times worse than for our own optimal model giving $S^{2} \simeq 0.6$. In addition, the inferred rotation period (assuming a solid body) is now about equal to $29500 \mathrm{~s}$, which is slightly but significantly shorter than the orbital period of 32486 s. Hence, rotational splitting in Feige 48 appears sufficiently important that the specific $m=0$ assignments make a difference in the derived structural parameters.

Inspired by these results showing that the two sets of mode identification discussed so far differ only through their $m$ values, we felt that another model with "intermediate" values of its merit function, structural parameters, rotation period and $m$ values could possibly exist. And indeed, another search led to a third possibility: a model whose characteristics are summarized in the middle column of Table 3 . In that case, the value $\left(S^{2} \simeq 0.9\right)$ of the merit function does not significantly differ from that of our initial optimal model, although the latter remains the best formal solution that we found. The inferred value of the rotation period of the intermediate model, $P_{\text {rot }} \simeq 30500 \mathrm{~s}$, is formally shorter than the orbital period but remains consistent with the idea of spin-orbit synchronism within the uncertainties. We note that the inferred total mass of this intermediate model is closer to the canonical value generally expected for a sdB star. It remains to be seen from time-resolved spectroscopy if $m$ mode discrimination is possible in Feige 48.

\section{Feige 48 , a solid-body rotator in a tidally locked binary system}

The present asteroseismic analysis, exploiting our new asteroseismic tools that incorporate the effects of star rotation, strongly suggests that Feige 48 is most likely a solid body rotator in synchronous rotation with its orbital companion. Our optimal model has a rotation period of $9.028 \mathrm{~h}( \pm 0.480 \mathrm{~h})$, which is essentially the same as the orbital period of the system $9.024 \mathrm{~h}( \pm 0.072 \mathrm{~h})$ determined independently by radial velocity variations ( $\mathrm{O}$ 'Toole et al. 2004). This is the first time that spin-orbit synchronism in a close binary system is demonstrated on the basis of 
Table 4. Inferred properties of Feige $48(V=13.46 \pm 0.02)$.

\begin{tabular}{cc}
\hline \hline Quantity & Estimated Value \\
\hline$T_{\text {eff }}(\mathrm{K})$ & $29580 \pm 370$ (spectroscopy) \\
$\log g$ & $5.462 \pm 0.006$ \\
$M_{*} / M_{\odot}$ & $0.519 \pm 0.009$ \\
$\log \left(M_{\text {env }} / M_{*}\right)$ & $-2.52 \pm 0.06$ \\
$P_{\text {rot }}(\mathrm{h})$ & $9.028 \pm 0.480$ \\
$R / R_{\odot}\left(M_{*}, g\right)$ & $0.221 \pm 0.003$ \\
$L / L_{\odot}\left(T_{\text {eff }}, R\right)$ & $33.8 \pm 2.7$ \\
$M_{V}\left(g, T_{\text {eff }}, M_{*}\right)$ & $3.89 \pm 0.06$ \\
$d\left(V, M_{V}\right)(\mathrm{pc})$ & $820 \pm 31$ \\
$V_{\text {eq }}\left(R, P_{\text {rot }}\right)\left(\mathrm{km} \mathrm{s}^{-1}\right)$ & $29.8 \pm 2.0$ \\
$i\left(^{\circ}\right)$ & $\$ 9.7 \pm 0.7$ \\
\hline
\end{tabular}

asteroseismic determinations of the rotation period and inner profile of the star. Unfortunately, the age of the sdB star cannot be inferred from our static models. According to the evolutionary tracks in a $\log g-T_{\text {eff }}$ diagram, Feige 48 is near the terminalage EHB (TAEHB), which corresponds to a typical time span of $\sim 100$ Myr since the zero-age EHB (ZAEHB). Hence, this appears to be sufficiently long for the complete synchronizing action of the tidal forces to have occurred in the sdB star. Such a result could provide new elements to the puzzling problem of tidal dissipation in radiative stellar envelopes of hot stars (see Zahn 1977 vs. Tassoul \& Tassoul 1992). It has to be said that little is known about the unseen white dwarf companion, and a fortiori about its level of synchronization. Theoretical investigations of Campbell $(1983,1984)$ have shown that white dwarfs have very long synchronization timescales (significantly longer than those in hot stars with radiative envelopes), except in the presence of strong magnetic fields. There is no hint of such fields in the system Feige 48, and the white dwarf component is probably not yet synchronized to its companion.

Along with the rotation properties, our analysis also leads to an updated determination of the structural parameters of the sdB star Feige 48, as summarized in Table 4. The primary parameters, i.e., those naturally derived from the optimization procedure, are $\log g, \log \left(M_{\text {env }} / M_{*}\right), M_{*}$, and $P_{\text {rot }}$, whereas $T_{\text {eff }}$ is determined from spectroscopy. The spectroscopic estimate of the surface gravity also helps to identify the appropriate asteroseismic solution among the duplicities, but once done, the $\log g$ parameter is derived with a much higher precision from asteroseismology. For its part, the hydrogen-rich envelope (a pure product of asteroseismology) is found to be rather thick, a result which is fully consistent with the correlation $T_{\mathrm{eff}}-\log q(\mathrm{H})$ as a function of total mass expected from stellar evolution theory. We note that our estimated value for the mass is somewhat higher than the canonical value of $\sim 0.48 M_{\odot}$ for subdwarf B stars. It also resides in the very upper range of allowable masses according to Han et al. $(2002,2003)$ for common envelope evolution, the most natural formation scenario in the case of a close binary system $\mathrm{sdB}+$ white dwarf such as Feige 48.

On the basis of these primary parameters, a set of secondary quantities can be derived: the stellar radius $R$ (as a function of $M_{*}$ and $g$ ), the luminosity $L$ (as a function of $T_{\text {eff }}$ and $R$ ), the absolute magnitude $M_{V}$ (as a function of $g, T_{\text {eff }}$ and $M_{*}$ in conjunction with the use of detailed model atmospheres) and the distance from Earth $d$ (as a function of $V$ and $M_{V}$ ). The equatorial rotation velocity $V_{\text {eq }}$ can also be calculated (as a function of $R$ and $P_{\text {rot }}$ ), and an upper limit on the inclination angle $i$ can be derived from the upper bound $V \sin i \lesssim 5 \mathrm{~km} \mathrm{~s}^{-1}$ measured in sharp metal lines seen in the spectrum. The evaluation of the internal
1- $\sigma$ errors follows the prescription of Charpinet et al. (2005a), a method used in the case where the number of modes in the pulsation spectra is of the same order as the number of free parameters necessary to specify the models. The uncertainties on secondary parameters are derived from the values obtained for the primary quantities. All these errors are statistical ones, with the understanding that the true uncertainties due to systematic effects are probably larger than the formal errors quoted here. Finally, we derive a revised estimate for the lower limit for the mass of the invisible companion to Feige 48, using the mass function

$f\left(M_{\mathrm{sdB}}, M_{\mathrm{comp}}\right)=\frac{M_{\mathrm{comp}}^{3}(\sin i)^{3}}{\left(M_{\mathrm{sdB}}+M_{\mathrm{comp}}\right)^{2}}=\frac{K_{\mathrm{sdB}}^{3} P}{2 \pi G}$

equal to $f\left(M_{\mathrm{sdB}}, M_{\text {comp }}\right)=0.000856 \pm 0.000019 M_{\odot}(\mathrm{O}$ 'Toole et al. 2004). Using the limit to the inclination angle $i$, it gives a minimum mass for the companion of $0.61 M_{\odot}$, a very typical value for a white dwarf.

\section{Conclusion}

We have presented a new asteroseismological analysis of the rapidly pulsating subdwarf B star Feige 48 using our latest period-matching codes that now incorporate the effects of stellar rotation on pulsation modes. This updated approach to sdB asteroseismology permits, when appropriate, the simultaneous fit of all the rotationally-split frequency components (a total of nine modes in the case of Feige 48). This constitutes a significant progress over the previous analysis of this star which was based only on the central $m=0$ components (four periods that had to be identified a priori). These new tools also allowed us to explore the impact of various internal rotation profiles on the pulsation period fits. Our most significant result is that Feige 48 most likely rotates as a solid body - ruling out in the process the idea of differential (and especially fast) core rotation - and that its rotation period $(9.028 \pm 0.480 \mathrm{~h})$ is virtually the same as the orbital period $\left(P_{\text {orb }}=9.024 \pm 0.072 \mathrm{~h}\right)$ measured through independent means by O'Toole et al. (2004). This provides an eloquent demonstration that a relatively close binary system such as Feige 48 has reached complete spin-orbit synchronism within its lifetime. This is the first time that asteroseismology has ever been used for such a demonstration.

Our approach has also led us to revise the asteroseismological estimates of the surface gravity $\log g$, the hydrogen-rich envelope thickness $\log \left(M_{\text {env }} / M_{*}\right)$, and the total stellar mass $M_{*}$ of Feige 48. We offer two possible new solutions as given in Table 3. In both cases, the inferred surface gravity value is entirely consistent by construction with spectroscopic estimates available from at least three independent sources and atmosphere modelings. The H-rich envelope is found to be rather massive, which is quite consistent with expectations from stellar evolution theory for a sdB star with such effective temperature and mass. The stellar mass itself for the best formal solution is found in the upper range allowed by Han et al. $(2002,2003)$ for the common envelope evolutionary channel, the most likely scenario to explain the formation of close sdB+WD systems like Feige 48. The mode identification inferred with this model - where positive $m$ values seems to be favored in two groups of multiplets has to be checked by independent means such as time-resolved spectroscopy. Another obvious follow-up study for Feige 48 is to test the values of the degree $\ell$ obtained in this study with either time-resolved spectroscopy or multicolor photometry, as was done successfully for other sdB pulsators (see, e.g., Randall et al. 2005; Tremblay et al. 2006 and; more recently, 
Charpinet et al. 2007). Finally, we note that future improvements in our abilities at building ever more realistic models of sdB stars may change somewhat the inferred values of the structural parameters of Feige 48. At the same time, however, we do not see how such improvements could seriously affect the main conclusion of this work regarding spin-orbit synchronism.

Acknowledgements. We thank the referee for his constructive comments. This work was supported in part by the NSERC of Canada. G.F. also acknowledges the contribution of the Canada Research Chair Program. This work made extensive use of the computing facilities offered by the Calcul en Midi-Pyrénées (CALMIP) project, France. Experiments presented in this paper were also carried out using the Grid'5000 experimental testbed, an initiative from the French Ministry of Research through the ACI GRID incentive action, INRIA, CNRS and RENATER and other contributing partners (see https://www . grid5000. fr).

\section{References}

Billères, M., Fontaine, G., Brassard, P., et al. 2000, ApJ, 530, 441 Brassard, P., Pelletier, C., Fontaine, G., \& Wesemael, F. 1992, ApJS, 80, 725 Brassard, P., Fontaine, G., Billères, M., et al. 2001, ApJ, 563, 1013

Brown, T. M., Ferguson, H. C., Davidsen, A. F., \& Dorman, B. 1997, ApJ, 482, 685

Campbell, C. G. 1983, MNRAS, 205, 1031

Campbell, C. G. 1984, MNRAS, 207, 433

Charpinet, S., Fontaine, G., Brassard, P., \& Dorman B. 1996, ApJ, 471, L103

Charpinet, S., Fontaine, G., Brassard, P., et al. 1997, ApJ, 483, L123

Charpinet, S., Fontaine, G., \& Brassard, P. 2001, PASP, 113, 775

Charpinet, S., Fontaine, G., \& Brassard, P. 2003, in White Dwarfs, NATO ASIB Proc., 105, 69

Charpinet, S., Fontaine, G., Brassard, P., et al. 2005a, A\&A, 443, 251

Charpinet, S., Fontaine, G., Brassard, P., Green, E. M., \& Chayer, P. 2005b, A\&A, 437, 575

Charpinet, S., Silvotti, R., Bonnano, A., et al. 2006, A\&A, 459, 565

Charpinet, S., Fontaine, G., Brassard, P., et al. 2007, in Proc. Third Meet. on Subdwarf O and B stars, ASPC, ed. U. Heber, R. Napiwotzki, \& C. S. Jeffery, in press

Chayer, P., Fontaine, G., Fontaine, M., et. al. 2004, Ap\&SS, 291, 359

Claret, A., \& Cunha, N. C. S. 1997, A\&A, 318, 187

Claret, A., Gimenez, A., \& Cunha, N. C. S. 1995, A\&A, 299, 724
D’Cruz, N., Dorman, B., Rood, R. T., \& O’Connell, R. W. 1996, ApJ, 466, 359 Dorman, B., Rood, R. T., \& O’Connell, R. W. 1993, ApJ, 419, 596 Fontaine, G., Brassard, P., Charpinet, S., et al. 2006a, ESA SP-624

Fontaine, G. Brassard, P., Charpinet, S., \& Chayer, P. 2006b, MmSAI, 77, 49

Geier, S., Nesslinger, S., Heber, U., et. al. 2007, A\&A, 464, 299

Geier, S., Nesslinger, S., Heber, U., et. al. 2008, A\&A, 477, L13

Green, E. M.. Liebert, J. W., \& Saffer, R. A. 1997, in The Third Conf. on Faint Blue Stars, ed. A. G. D. Philip, J. Liebert, R. Saffer, \& D. S. Hayes, 417

Han, Z., Podsiadlowski, P., Maxted, P. F. L., Marsh, T. R., \& Ivanova, N. 2002, MNRAS, 336, 449

Han, Z., Podsiadlowski, P., Maxted, P. F. L., \& Marsh, T. R. 2003, MNRAS, 341, 669

Heber, U. 1986, A\&A, 155, 33

Heber, U., Reid, I. N., \& Werner, K. 1999, A\&A, 348, L25

Heber, U., Reid, I. N., \& Werner, K. 2000, A\&A, 363, 198

Hilditch, R. W. 2001, An introduction to Close Binary Stars, The Observatory, 121,389

Jeffery, C. S., \& Saio, H. 2006, MNRAS, 372, L48

Jeffery, C. S., \& Saio, H. 2007, MNRAS, 378, 379

Kawaler, S. D., \& Hostler, S. R. 2005, ApJ, 621, 432

Kilkenny, D., Koen, C., O’Donoghue, D., \& Stobie, R.S. 1997, MNRAS, 285, 640

Koen, C., O’Donoghue, D., Pollaco, D. L., \& Nitta, A. 1998, MNRAS, 300, 1105

Maxted, P. F. L., Marsh, T. R., \& North, R. C. 2000, MNRAS, 317, L41

Maxted, P. F. L., Heber, U., Marsh, T. R., \& North, R. C. 2001, MNRAS, 326, 1391

Morales-Rueda, L., Maxted, P. F. L., Marsh, T. R., Kilkenny, D., \& O’Donoghue, D. 2006, Baltic Astron., 15, 187

Napiwotzki, R., Karl, C. A., Lisker, T., et al. 2004, Ap\&SS, 291, 321

Orosz, J.-A., \& Wade, R. A. 1999, MNRAS, 310, 773

O’Toole, S. J., \& Heber, U. 2006, A\&A, 452, 579

O'Toole, S. J., Heber, U., \& Benjamin, R. A. 2004, A\&A, 422, 1053

Randall, S. K., Fontaine, G., Brassard, P., \& Bergeron, P. 2005, ApJS, 161, 456

Randall, S. K., Fontaine, G., Charpinet, S., et al. 2006, ApJ, 648, 637

Randall, S. K., Green, E. M., Van Grootel, V., et al. 2007, A\&A, 476, 1317

Reed, M. D., Kawaler, S. D., Zola, S., et al. 2004, MNRAS, 348, 1164

Saffer, R. A., Green, E. M. \& Browers, T. 2001, in 12th European Workshop on White Dwarfs, ed. J. L. Provencal, H. L. Shipman, J. MacDonald, \& S. Goodchild, ASP Conf. Ser., 226, 408

Tassoul, J.-L., \& Tassoul, M. 1992, ApJ, 395, 259

Tremblay, P. E., Fontaine, G., Brassard, P., et al. 2006, ApJS, 165, 551

Zahn, J.-P. 1977, A\&A, 57, 383 